\title{
Stellar Rotation in the Young Cluster M17
}

\author{
Wenjin Huang and Douglas Gies \\ Georgia State Univ., Physics \& Astronomy, Atlanta, GA 30303 U.S.A. \\ Philip Massey \\ Lowell Observatory, 1400 W. Mars Hill, Flagstaff, AZ 86001, U.S.A.
}

Peter S. Conti

University of Colorado, JILA, Box 440, Boulder, CO 80309 U.S.A.

\begin{abstract}
We constructed line profile models for young OB stars in M17 using TLUSTY and SYNSPEC, including the effects of rotational flattening and gravitational darkening. The values of $v \sin i$ for 8 member stars (with spectral classifications $04.5 \mathrm{~V}$ to $09 \mathrm{~V}$ ) range from $7 \mathrm{~km} \mathrm{~s}^{-1}$ to $168 \mathrm{~km} \mathrm{~s}^{-1}$. The mean value of $v \sin i\left(102 \mathrm{~km} \mathrm{~s}^{-1}\right)$ is lower than expected for very young clusters.
\end{abstract}

\section{Introduction and Observations}

M17 is one of the most massive molecular clouds and most luminous HII regions in the Galaxy, and its member stars are in their formation stage (Hanson, Conti, \& Rieke 1996). Major investigations on M17 have been done only in radio and IR due to the large extinction caused by the thick surrounding medium (Bumgardner 1992; Hanson, Howarth, \& Conti 1997, hereafter HHC). However, there are some regions that do have relatively low opacities so that YSOs can be studied at optical wavelengths. Fifteen very young OB stars have recently been identified in M17 (HHC). Some of these show evidence of circumstellar disks, while others appear to have cleared out their surroundings. We can determine the spectral type and projected rotational velocity of these young stars using spectrum synthesis techniques.

We observed the M17 stars during a single observing run (2000 July 1116) using the WIYN 3.5-m/Hydra multi-object spectrograph with the 860@30.9 grating in second order for a resolving power $R=5700$. We obtained spectra in the red (5000-5900 $\AA$ ) because the targets are significantly brighter in $V$ than $B$. We obtained spectra of 15 young OB stars, six of which have a known disk morphology (HHC).

\section{Projected Rotational Velocities}

Using TLUSTY and SYNSPEC (Hubeny \& Lanz 1995), we built a grid of intensity line profiles parameterized by $T_{\text {eff }}, \log g$ and $\mu$ (in this paper, we assume solar abundances for simplicity) with non-LTE treatments. The synthesis of line 
profiles for a rotating star is based on this grid. We investigated several spectral lines in the observed waveband, but found that only He II $\lambda 5411$ gave reliable measurements of $v \sin i$ for 8 member stars. The results are listed in Table 1 . Interestingly, all the stars with disk morphology in our investigation (except B289) have no measurable lines in their red spectra.

Table 1. Spectral Classifications and Projected Rotational Velocities

\begin{tabular}{ccccc}
\hline \hline Name & $\begin{array}{c}\text { Sp. Type } \\
(\text { HHC })\end{array}$ & $\begin{array}{c}\text { Sp. Type } \\
\left(W_{\lambda}(\text { He II } \lambda 5411)\right)\end{array}$ & $\begin{array}{c}v \sin i \\
\left(\mathrm{~km} \mathrm{~s}^{-1}\right)\end{array}$ & Notes \\
\hline OI345 & O6 V & O6.5 V & 106 & SB1 \\
B098 & O9 V & O8.5 V & 79 & \\
B111 & O5 V & O4.5 V & 168 & \\
B164 & O7-O8 V & O6 V & 59 & SB2? \\
B189 & O5 V & O5 V & 143 & \\
B260 & O7-O8 V & O7.5 V & 114 & \\
B289 & O9.5 V & O9 V & 139 & Disk \\
B311 & $\ldots$ & O8.5 V & 7 & \\
\hline \hline
\end{tabular}

The mean value of $v \sin i\left(102 \mathrm{~km} \mathrm{~s}^{-1}\right)$ is lower than the expected for OB stars in very young clusters $\left(150-200 \mathrm{~km} \mathrm{~s}^{-1}\right.$; Howarth et al. 1997). Although our sample of 8 member stars is small, we doubt that this low mean can be explained by systematically low inclination angles. There may be some efficient braking mechanism that has slowed the rotation of these stars. It is possible that the angular momentum of the natal cloud was preferentially deposited in an enhanced population of binaries in M17. However, our one-week observing run is not long enough to find binary systems with medium and long periods, and additional observations will be required to determine the actual incidence of binaries.

\section{References}

Bumgardner, T. E. 1992, M. S. thesis, Ohio State University

Hanson, M. M., Conti, P. S., \& Rieke, M. J. 1996, ApJS 107, 281

Hanson, M. M., Howarth, I. D., \& Conti, P. S. 1997, ApJ 489, 698

Howarth, I. D., Siebert, K. W., Hussain, G. J., \& Prinja, R. K. 1997, MNRAS 284, 265

Hubeny, I., \& Lanz, T. 1995, ApJ 439, 875 